\title{
Criminologie
}

\section{De la criminalisation à la légalisation des drogues : de Charybde en Scylla?}

\section{Line Beauchesne}

Volume 22, numéro 1, 1989

Drogues et criminalités

URI : https://id.erudit.org/iderudit/017274ar

DOI : https://doi.org/10.7202/017274ar

Aller au sommaire du numéro

Éditeur(s)

Les Presses de l'Université de Montréal

ISSN

0316-0041 (imprimé)

1492-1367 (numérique)

Découvrir la revue

Citer cet article

Beauchesne, L. (1989). De la criminalisation à la légalisation des drogues : de Charybde en Scylla ? Criminologie, 22(1), 67-83.

https://doi.org/10.7202/017274ar
Résumé de l'article

If the drugs now considered illegal were legalized, what difficulties would have to be resolved? To answer this question, this article first points out the limitations of a policy of decriminilization in the case of soft drugs; it then shows that today, legalization and marketing of legal drugs go hand in hand and that this is a dangerous model for the legalization of drugs.

Hence although the need to legalize all drugs in order to alleviate the present problems caused by their criminalization is easy enough to demonstrate, to find a model of legal distribution of drugs that avoids the negative consequences of the present policies regarding legal drugs is much more difficult. It merits some thought. 
If the drugs now considered illegal were legalized, what difficulties would have to be resolved? To answer this question, this article first points out the limitations of a policy of decriminilization in the case of soft drugs; it then shows that today, legalization and marketing of legal drugs go hand in hand and that this is a dangerous model for the legalization of drugs.

Hence although the need to legalize all drugs in order to alleviate the present problems caused by their criminalization is easy enough to demonstrate, to find a model of legal distribution of drugs that avoids the negative consequences of the present policies regarding legal drugs is much more difficult. It merits some thought.

Les commissions d'enquête de différents pays et plusieurs chercheurs qui se sont penchés sur les lois en matière de drogues et leur application en arrivent à la même conclusion : les lois sur les drogues, à l'heure actuelle, causent plus de torts que les drogues elles-mêmes (Bertrand, 1986:187). Est-ce à dire que si on légalisait les drogues actuellement illégales, ne resteraient à régler que les problèmes liés à leur consommation?

Ce schéma, bien sûr, est simpliste. Mais lorsqu'on veut tenter de le dépasser, ce n'est pas si facile. Que répondre aux intervenants qui oeuvrent auprès de personnes à la consommation d'alcool et/ou de médicaments jugée problématique, pour calmer leurs craintes de voir de nouveaux produits à l'origine de toxicomanies devenir plus aisément disponibles par leur légalisation? Quelle situation en matière de distribution de drogues convient-il d'envisager dans un contexte légal moins répressif que celui qui prévaut maintenant?

C'est une réflexion dans cette direction que je veux amorcer ici, pour ceux qui se préoccupent non pas de condamner ceux qui prennent des drogues, mais de les comprendre et même de les aider s'ils en font la demande.

Dans un premier temps, je rappellerai les effets néfastes de la criminalisation actuelle en matière de drogues et $\mathrm{j}$ 'indiquerai les limites d'une politique de décriminalisation des «drogues douces» pour corriger ces effets. Dans un deuxième temps, je soulignerai les retombées

* L'auteure est professeure au Département de criminologie, Université d'Ottawa, 75, av. Laurier, Ottawa, Ontario, K1N 6N5. 
positives et négatives qu'il faut envisager dans l'éventualité de la légalisation des drogues, selon les changements qui accompagneraient cette modification des lois.

\section{I. -- CRIMINALISATION/DÉCRIMINALISATION}

\section{A) CRIMINALISATION : LA SITUATION ACTUELLE}

Hulsman et van Ransbeek (1983) distinguent deux types de problèmes qui peuvent être engendrés par les drogues. Il y a les problèmes primaires qui sont spécifiquement liés à l'usage des drogues, aux dynamiques personnelles que les gens établissent avec ces produits, dynamiques dont les retombées se répercutent parfois négativement sur leur entourage. Il y a également les problèmes secondaires qui sont liés à la politique légale des drogues, principalement à l'interdiction des stupéfiants. Laissons pour le moment les problèmes primaires à l'écart et abordons les problèmes secondaires; ceux-ci sont relativement aisés à identifier, puisqu'une bonne partie de ces problèmes constituent la répétition du scénario qui se joua lors de la prohibition de l'alcool aux États-Unis pendant les années 20.

La première évidence est que l'illégalité de certaines drogues n'a pas diminué le nombre de consommateurs de ces produits au Canada, mais a augmenté le nombre de personnes criminalisées, surtout chez les jeunes (Smart, 1983; Bertrand, 1984). La curiosité étant l'un des facteurs majeurs d'essai des drogues (Brisson et al., 1987), leur caractère illégal devient, chez plusieurs, un facteur stimulant l'essai de ces produits et chez certains, encourage leur consommation et ce, même dans le cas de l'héroïne (de Jong, 1983). Boire de l'alcool ou fumer un joint, ce n'est pas seulement choisir un effet: "La préférence donnnée à une certaine drogue est liée à la valeur qu' on lui donne, à la signification et à la fonction qu' elle doit remplir dans le groupe et à l'effet qu'elle produit (Hulsman et van Ransbeek, 1983:271). Il y a longtemps que les publicitaires ont compris cela dans la promotion des drogues légales. Chez les compagnies de bière, dans les messages commerciaux, on ne compétitionne pas tant sur le goût de la bière que sur l'image qu'elle véhicule: la bière du sportif, des femmes d'affaires, du séducteur, etc. On ne boit pas seulement une bière, on boit un statut social (Germain, 1984). L'image et la fonction jouent également un rôle dans la consommation des drogues illégales (Brisson et al., 1987).

La consommation des drogues illégales a toutefois ceci de particulier: elle marginalise plus ou moins fortement les consommateurs à 
cause de la menace légale de sanction. Il y a d'un côté ceux qui consomment des drogues illégales, et de l'autre ceux qui n'en consomment pas (Laplante, 1974). Cette marginalisation rend difficile l'intervention en cas de problèmes liés à la consommation de drogues illégales, car les organismes institutionnels, ou même les amis ou parents qui pourraient apporter support et aide au consommateur sont souvent de l'autre côté du mur, parmi ceux qui n'ont pas été «initiés», qui jugent de loin, qui ne comprendront pas. Cette marginalisation crée une méfiance qui diminue les possibilités d'aide psycho-sociale au moment où elle aurait été nécessaire alors que, devenues toxicomanes, les personnes se font remarquer par un état de détérioration physique et mentale et de dégradation sociale avancé (voir à ce sujet les témoignages éloquents recueillis par Hermann et Rieck, 1981).

Comme lors de la prohibition de l'alcool, un marché illégal des produits interdits s'est développé. Sur le marché noir, les prix et la qualité ne sont pas contrôlés. En fonction des risques, de la demande et des intermédiaires, les règles du jeu dans le trafic des drogues peuvent engendrer corruption et violence, même chez les policiers et les gardiens de prison, car les possibilités de profits sont très importantes. Cercle vicieux : à cause de cette corruption et de cette violence, certains recommandent le renforcement des lois, ce qui augmente les risques pour les trafiquants, fait monter les prix et les possibilités de profits, et... la violence et la corruption.

Ce cercle ne peut être rompu par l'arrestation des trafiquants; ils sont beaucoup trop puissants (Glorie, 1983; Mc Nicoll, 1984; Lamarche, 1985; Rico, 1986). Il n'y a qu'à jeter un coup d'oeil aux statistiques canadiennes du Bureau des drogues dangereuses pour constater que les trafiquants sont peu touchés par les arrestations et les sanctions; la clientèle des arrestations est composée principalement de jeunes $(85 \%)$, pour possession $(80 \%)$, surtout de cannabis $(93 \%)$ (chiffres rapportés par Bertrand, 1984). Cette criminalisation de milliers de gens (plus de 26 000/année au Canada : Bertrand, 1984) entraîne non seulement des coûts énormes et du temps inutilement gaspillé par l'appareil pénal, la police, les avocats, etc., mais stigmatise les personnes criminalisées, même pour simple possession de cannabis (Erickson, 1980). Cela signifie que la criminalisation en matière de drogues au Canada a produit une forte répression (des pouvoirs extraordinaires de fouille et de perquisition furent donnés à la police au nom de la lutte contre la drogue) sans prévenir les toxicomanies (Beauchesne, $1988 \mathrm{a}, \mathrm{b})$. 
Non seulement cette répression et cette violence n'ont-elles pas diminué le nombre de consommateurs de drogues illégales, mais il semble bien que la mauvaise qualité des drogues sur le marché noir ait été à l'origine de maintes intoxications, de troubles graves pour la santé et même de morts de consommateurs de drogues. De plus, les prix élevés de plusieurs drogues illégales ont forcé certains usagers à se procurer illégalement l'argent nécessaire à leur consommation, surtout lorsque des dynamiques de dépendance se sont établies.

Morts, corruption, violence, criminalité, répression, coûts inutiles et temps gaspillé par le système de justice pénale, voilà les problèmes secondaires liés à la politique actuelle en matière de drogues. Il est clair que cette politique crée plus de problèmes sociaux qu'elle n'en règle. Les diverses commissions d'enquête gouvernementales sur la question ont admis cela (Bertrand, 1986:187). Devant ces constatations, toutefois, peu ont clamé bien haut une volonté de légaliser toutes les drogues; en dehors du statu quo prudent politiquement, c'est la décriminalisation des «drogues douces» qui reçoit le plus d'adeptes.

\section{B) LA DÉCRIMINALISATION DES «DROGUES DOUCES»}

La décriminalisation des «drogues douces» est un moyen important de diminution de la répression à l'égard de milliers de personnes arrêtées chaque année pour simple possession de cannabis au Canada. Par ces milliers d'arrestations, il est clair qu'on n'a pas diminué l'usage du cannabis, et qu'il y a lieu de s'interroger sur l'application sélective de la Loi sur les stupéfiants en regard des 4,5 millions d'usagers plus ou moins réguliers de cannabis au Canada (Bertrand, 1984). On arrête quelqu'un pour possession simple de cannabis quand il dérange et qu'on veut lui faire peur pour qu'il entre dans la norme; quand on le soupçonne d'un autre délit sans preuve; quand on a besoin d'indicateurs, etc. La logique de ces arrestations tient plus lieu d'instrument de contrôle par la police que de prévention des toxicomanies. Pour cette raison, d'ailleurs, il ne faut pas croire que les 26000 jeunes arrêtés au Canada pour simple possession de cannabis ne le seront plus s'il y a décriminalisation des «drogues douces». Disons simplement que les jeunes seraient moins aisément arrêtés sans ce prétexte d'usage de drogues illégales; la police a d'autres ressources mais elles n'ont pas cette ampleur. C'est pourquoi la décriminalisation des «drogues douces» constitue une mesure appréciable de diminution des mécanismes de répression abusifs.

L'autre aspect important est la possibilité de sortir de la marginalité et de la clandestinité pour les usagers de «drogues douces»; de 
manière relativement restreinte, toutefois. Décriminaliser ne signifie pas légaliser, c'est-à-dire assurer des contrôles de la qualité et des prix des produits en permettant des sources d'approvisionnement adéquates. Dans son rapport sur le cannabis, la Commission Le Dain en recommandait la décriminalisation parce qu'elle reconnaissait la nocivité mineure du produit; néanmoins, elle refusait sa légalisation parce qu'elle ne voulait pas (ou n'osait pas) cautionner des comportements allant à l'encontre des normes socio-économiques de production, tel l'usage récréatif de drogues:

bien que nous puissions dire que l'usage des psychotropes à des fin non médicales doive être condamné en principe...

\section{(...)}

Les effets de certains usages de la drogue sur la société constituent aussi une source de préoccupation. Ils comprennent: le danger que présente pour d'autres un comportement influencé par la drogue, comme la violence ou une diminution de rendement (...) (Commission Le Dain, 1972:267-268).

Bref, les impératifs de production ont prévalu sur la logique. En effet, pourquoi décriminaliser sans légaliser? Agir ainsi c'est faire en sorte que les usagers doivent continuer à s'approvisionner sur le marché noir. Pourquoi maintenir dans l'illégalité un produit dont on s'accorde à dire qu'il n'est pas nocif lorsqu'il est de qualité?

La commissaire Bertrand a répondu à cette recommandation de la Commission Le Dain en proclamant sa dissidence (Commission Le Dain, 1972:303-311). Elle explique que c'est un non-sens de permettre la vente de l'alcool et pas du cannabis étant donné que l'usage du cannabis est déjà très répandu. Si on veut prévenir les intoxications et les toxicomanies, dit-elle, il est nécessaire qu'il y ait un contrôle de la qualité par la légalisation du cannabis, qualité qui «permettrait à l'usager de mieux connaître les effets à escompter et d'apprendre à doser sa consommation en conséquence (305). Il est nécessaire également qu'il $\mathrm{y}$ ait une meilleure information sur les produits et une éducation adéquate en matière de santé. Ce n'est pas en maintenant les sources d'approvisionnement illégales et de mauvaise qualité, en obligeant l'usager à se marginaliser dès qu'il se procure du cannabis, que nous préviendrons les toxicomanies, explique Bertrand. Par contre, le meilleur moyen de faire perdre leur crédibilité aux lois, est de permettre l'usage de certaines drogues à l'encontre des autres.

Dans ce débat sur la possibilité de décriminaliser les «drogues douces», il est utile de revenir sur un élément trop souvent négligé : la 
catégorisation des drogues en «drogues douces» versus les «drogues dures» et le fait que ceux qui sont en faveur de la décriminalisation ou de la légalisation des «drogues douces» uniquement, le sont généralement à cause de leur croyance en la faible nocivité des produits concernés. Cette catégorisation des drogues fausse le débat sur la légalisation des drogues en camouflant deux choses:

- la première est que ce sont des produits étrangers à l'économie américaine qui ont été interdits (les stupéfiants sont des produits interdits internationalement) et non des produits plus dangereux que les drogues légales; il est bon de rappeler que les États-Unis sont le maître d'oeuvre de la classification légale des drogues et ce, à l'échelle internationale (Helmer, 1975; Glorie, 1984). Si c'est seion leur effet que l'on veut classer les produits, et si ce n'est que la possibilité d'engendrer des dépendances suite à un mauvais usage qui les qualifie de «drogues dures», on peut considérer, entre autres, les médicaments, le sucre, le tabac et l'alcool comme des «drogues dures». Pourquoi ces produits ne sont-ils pas illégaux? Ou encore, pourquoi peut-on gérer l'usage de ces produits et pas celui des drogues dites dures qui sont illégales, si on peut garantir la qualité des produits et de l'information sur leur usage?

- la deuxième découle de la première. Cette classification sousentend que c'est l'effet qui caractérise la nocivité du produit indépendamment du type d'usage. Ne bouffer que du sucre ou des valiums est certainement très nocif. Et alors? Il s'agit de faire usage de ces produits de manière adéquate pour sa santé et son mode de vie en général; la nocivité des produits vient de leur mauvais usage. Alors comment classer les drogues? Le classement des drogues en fonction de leur nocivité ne peut être très utile car ce genre de classification dépend des critères subjectifs utilisés pour définir la nocivité et l'usage qui est fait des produits (Campbell, in Commission Le Dain, 1973:248-249).

En somme, la solution de décriminaliser les drogues dites douces, même si elle a l'avantage de diminuer les contrôles policiers sur certaines clientèles d'usagers et si elle permet une certaine démarginalisation des consommateurs de ces drogues, demeure une solution limitée. Elle n'amène pas de contrôle de qualité et de meilleurs circuits de distribution des produits qui permettraient la diminution des intoxications; elle ne légitime pas l'usage récréatif de drogues; elle ne considère pas l'usager de drogues capable de gérer sa consommation, même s'il est adéquatement informé sur les produits, sauf pour des drogues où les possibilités de dépendances sont presque nulles; elle ne remet pas en 
question la politique actuelle en matière de drogues avec tous les problèmes secondaires qui en découlent.

Tout ceci m'amène à la conclusion que, non seulement ce sont toutes les drogues qu'il faut légaliser (y inclus l'héröne) pour contrer les effets négatifs de la criminalisation, de la marginalisation, de la mauvaise qualité des produits et de leur prix élevé sur le marché noir, mais il faut le faire dans une optique qui remette même en question la politique actuelle à l'égard des drogues légales pouvant également engendrer des toxicomanies; c'est le seul moyen d'arriver à construire une information et de faire une éducation sur les psychotropes susceptible d'augmenter les capacités de choix et d'autonomie des gens à l'égard de ces produits.

Toutefois, la décriminalisation du cannabis et de ses dérivés, inutile si elle a pour objectif de prévenir les toxicomanies, demeure un moyen important de diminution de certains contrôles policiers abusifs.

\section{II. - LÉGALISATION}

Quelles seraient les conséquences d'une éventuelle légalisation des drogues?

La première a été mentionnée plus haut: une diminution de la répression policière. Cela entraînerait également une diminution de la corruption policière occasionnée par les profits énormes réalisés sur le marché noir des drogues. La deuxième conséquence serait l'urgence d'une double action: d'une part, une éducation qui permette de mieux contrôler l'usage des psychotropes, d'autre part, un examen critique du droit d'intervention sociale et médicale que les interdits antérieurs avaient cautionné. La troisième conséquence serait la nécessité de redéfinir toute la dynamique commerciale sur les drogues.

\section{A) UN RENOUVELLEMENT DU SAVOIR SUR LES DROGUES}

Bâtir une éducation adéquate en matière de drogues pour prévenir les toxicomanies est une tâche peu aisée, entre autre, parce que notre savoir est très pauvre sur ces questions. Les études scientifiques sur les drogues, en particulier celles provenant du champ médical, se sont, en grande partie, moulées sur les normes de la loi; ces études sont venues camoufler dans la prétendue neutralité scientifique les enjeux de cette classification. En termes strictement méthodologiques, on retrouve dans 
nombre de ces études des préjugés que l'on confirme selon les besoins de la norme institutionnelle, un peu comme on classifie les médicaments selon la perception sociale de la maladie à combattre (Dagognet, 1984). Par exemple, Robins et al.(1980) constataient qu'aucune étude sur les effets de l'héroïne ne portait sur une clientèle autre qu'institutionnalisée pour consommation très problématique; ces études tiraient des conclusions générales sur les effets néfastes de l'hérö̈ne pour l'ensemble de la population. C'est prétendre décrire les effets de l'alcool de qualité sur la population à partir des séquelles sur des clochards qui consomment quotidiennement de l'alcool frelaté sans ingérer une nourriture adéquate pour leur santé. Méthodologiquement inacceptable, cette manière de faire est, dans le cas des drogues, devenue acceptable; il faut reconnaître que ces études servent bien les préjugés institutionnels négatifs à l'égard de ces produits.

Dans d'autres études, on a vérifié l'effet de l'absorption d'un produit à partir de quantités ingérées, de fréquences de consommation, et d'un environnement qui n'ont rien à voir avec les habitudes générales de consommation de ce produit. Par exemple, quelle est l'utilité de savoir que de fumer un joint est 3 fois plus nocif pour les poumons que de fumer une cigarette quand les habitudes de consommation des usagers de cannabis n'ont généralement rien à voir avec les habitudes de consommation des usagers de la cigarette? De toute façon, on peut dire de n'importe quel produit qu'il est nocif pour la santé s'il est absorbé quotidiennement à haute dose au détriment d'une alimentation saine. Avaler $2 \mathrm{~kg}$ de chocolat par jour pendant un an mène inévitablement à des troubles de santé graves. En prouvant scientifiquement cela, qu'estce qu'on a dit? Que le chocolat est dangereux et qu'il faut l'interdire parce qu'à haute dose il est nuisible pour la santé? C'est ce que se permettent de conclure un certain nombre d'études sur les drogues illégales:

Pour qu' un débat ou des recherches portant sur la "toxicité» du produit soient développés par les médecins, il faut que préalablement s'exprime une volonté politique d'invalidation. Cette volonté n'est généralement pas exprimée en tant que telle, elle reste donc en dehors de la discussion entre spécialistes mais réapparaît alors jusque dans le travail expérimental lui-même et sous différentes formes. C'est le cas par exemple des recherches menées par Georges Nahas qui s'acharne à démontrer scientifiquement la "toxicité» du cannabis (pour qu' une telle recherche se développe, encore faut-il que la question soit posée) et qui attribue en fin de compte au produit toutes les caractéristiques liées à l'image du toxicomane. Qui plus est, se faire une "spécialité» et construire son prestige dans la démonstration du danger que représente la consommation d' une substance conduit à relativiser la "nocivité» 
d'autres substances; dans ce domaine aussi le discours tenu dépend du rapport à l'objet de recherche. La valorisation de l'objet de recherche (ici contenu dans la maximisation du danger social qu'il représente) est un effet habituel des stratégies de mise en valeur des chercheurs. (Zafiropoulos et Pinell, 1982:73)

Reprenons l'exemple du chocolat. Si certaines personnes consomment des quantités telles de chocolat qu'elles se détruisent, on ne cherche pas la cause dans le chocolat, mais dans les motivations qu'elles ont à s'auto-détruire par le chocolat. De la même manière, si certaines personnes consomment des drogues de manière auto-destructrice, il y a lieu de s'interroger non pas sur les drogues, mais sur les motivations à les consommer de manière risquée pour leur vie (Valleur, 1984). La consommation destructrice de drogues, lorsque les risques sont pris volontairement, ne va pas disparaître avec la légalisation de celles-ci, pas plus que ne vont disparaitre les quartiers pauvres et les gens mal aimés, les adaptations difficiles aux conditions de vie, etc. L'erreur est de croire que les produits sont responsables des motivations à consommer, qu'il s'agit de faire disparaître les produits de l'environnement pour solutionner tous les problèmes. Par exemple, ce document de formation sur les drogues destiné aux services policiers qui explique que la consommation de marijuana est à l'origine de la contestation des valeurs des années soixante; la logique de ce raisonnement est donc de combattre l'usage de la marijuana pour faire disparaître la contestation:

Il est fascinant de méditer sur la culture à laquelle a donné naissance l'usage de la marijuana; le retour à la terre, l'existence méditative, la vie tribale, communautaire et le rejet de toute autorité traditionnelle et des institutions qui en découlent. Cette vision idyllique et pastorale a exercé un attrait quasi hypnotique sur de nombreux intellectuels de notre temps. Cette culture née de la drogue est voilée dans un tissu de mysticisme et de marge totalement dépourvu de la moindre parcelle consciente à l'effet que son attrayante philosophie prônant une évidente paix intérieure plus profonde, découle directement du moins en partie, des effets de la marijuana sur le cerveau. (Mc Nicoll, 1978:7)

Ce raisonnement, en inversant les motivations à consommer pour les présenter comme des effets des drogues, permet d'éviter de remettre en question les conditions de vie et les normes sociales qui amènent certaines personnes à s'auto-détruire, ou encore à fuir la réalité par l'usage des drogues. On préfère considérer ces personnes comme délinquantes à punir ou à guérir, sans remettre en cause le monde dans lequel elles vivent. Et pourtant, elles cherchent simplement à s'adapter au monde actuel ou à ne pas y étouffer. Les drogues illégales jouent, à ce titre, le même rôle que l'alcool et les médicaments : 
(...) la drogue ou la substance chimique elle-même n'est pas le problème mais bien le contexte psycho-social dans lequel elle circule.

(...)

peut-on concevoir la révolution industrielle et la dégradation psychique générale qu'elle imposa sans le secours de l'alcool? Peuton concevoir l'Europe, l'URSS et l'Amérique sans alcool? Les classes ouvrières et les petites bourgeoisies n'auraient jamais accepté ou traversé l'effarante déshumanisation de l'industrialisme sans l'amortissement psychique de l'alcool. Peut-on concevoir la vie moderne et l'angoisse qu'elle engendre chez des millions et des millions de gens sans l'aide des dépresseurs ou calmants genre Valium? (Georges Khal, in Hermann et Rieck, 1981:277-278)

Bref, tout notre savoir est à réorganiser en ce qui concerne les drogues, légales et illégales, pour construire une information adéquate et dispenser une éducation sur les drogues qui prévienne certaines toxicomanies, et mène même à la reconnaissance de la nécessité de changer certaines conditions psycho-sociales de vie (par exemple les polyvalentes au secondaire, cf. Hermann et Rieck, 1981).

Le renouvellement de notre savoir sur les drogues, dans un contexte de légalisation de tous les produits, demanderait également une redéfinition des pratiques socio-médicales actuelles, de même qu'une remise en question des droits d'intervention que certains services sociaux, médicaux et correctionnels se sont donnés dans le domaine des drogues.

B) UNE REDÉFINITION DES DROITS ET DES PRATIQUES D'INTERVENTION

Les pratiques des services sociaux et médicaux se distancient peu de la définition des problèmes telle que posée par les normes juridiques en matière de drogues (Érickson, 1985; Vertongen, 1986; Brisson, 1986; Glorie, 1984). La légalisation des drogues en redéfinissant les normes, nécessiterait donc une redéfinition de certains droits et pratiques d'intervention.

Au Canada, les intérêts corporatistes de la profession médicale se sont découpés dans leur formation et leurs pratiques en regard des autres professions qui veulent «guérir», par leur habilité à prescrire des médicaments; cette habileté leur a valu la couverture par l'assurancemaladie des actes médicaux qu'ils posent, financés par l'État, contrairement aux psychologues ou autres intervenants sociaux. Ainsi, les problèmes de santé ne sont plus reliés aux conditions de vie à changer, 
mais à un dérèglement aléatoire de l'organisme qui se corrige par des médicaments. Les gens ont désappris à prendre en charge leur corps et leur santé et réclament la pilule-miracle:

C'est dire que face à des problèmes liés à l' existence et aux conditions de vie contemporaines - stress, dépression, insomnie, troubles nerveux, mésadaptations de toutes sortes y compris certains comportements toxicomanes -, l'institution médico-thérapeutique occidentale, soutenue et pressée en cela par l'industrie pharmaceutique multinationale, préconise une intervention correctrice au niveau de la bio-chimie du cerveau, plutôt que des formes d'actions individuelles et collectives, visant le refus ou la transformation des conditions d'existence souvent à la source véritable de nos «malaises de civilisation». (Brisson, 1986:78)

En outre, pour les institutions publiques, les critères d'attribution des subventions poussent celles-ci à se modeler aux normes juridiques.

En somme, toute une logique économique de fonctionnement des institutions et des pratiques médicales permet de poursuivre de manière très rentable la surprescription des médicaments sans que cela fasse scandale, et disqualifie l'usage (problématique ou non) des drogues illégales. La définition des problèmes, des causes, l'évaluation des réussites et des échecs, ne seront pas les mêmes selon que l'on parle de drogues illégales ou légales pour répondre aux normes socio-économiques de la loi. Le meilleur exemple de cela est le mandat même de la Commission Le Dain $(1972,1973)$ : "Commission d'enquête sur l'usage des drogues à des fins non médicales». Comme si les drogues illégales ou non prescrites ne pouvaient être utilisées adéquatement par les usagers : on évite ainsi de contester le monopole du discours sur la santé que veut conserver la profession médicale (Bertrand, 1986 b). Comme si, également, l'usage des drogues prescrites était adéquat pour la santé par opposition à l'usage des drogues non prescrites: on évite ainsi de constater qu'il y a beaucoup plus de gens qui sont dépendants et même meurent à l'heure actuelle d'un mauvais usage des drogues prescrites plutôt que des drogues non-prescrites (Brisson, 1986; Sheskin, 1978). Ce découpage du problème à résoudre camoufle les enjeux des normes et restreint le débat à l'intérieur de celles-ci. Grâce à ce monopole de la prescription des médicaments, les médecins ont pu se construire un monopole dans le discours sur la santé qui sert les pouvoirs économiques :

La prétention à occuper une place hégémonique en matière de santé conduit les médecins à assurer leur contrôle sur certaines pratiques sociales les plus quotidiennes (élevage des enfants, pratiques sexuelles, hygiène, alimentation, boissons, sports, etc.); les 
modalités de ce contrôle peuvent rencontrer l'intérêt des groupes sociaux dominants à réglementer les moeurs et le mode de vie des dominés. (Zafiropoulos et Pinell, 1982:71)

Ainsi, dans une société où les drogues seraient légales, les médecins perdraient ce monopole si une meilleure éducation permettait aux gens de se réapproprier leur corps, leur santé, leur mode de vie. Les médecins devraient réajuster leur savoir et leurs pratiques en conséquence; ils ont d'ailleurs compris cela et ont déjà manifesté fortement leur opposition à la légalisation des drogues (Bertrand, $1986 \mathrm{a}, \mathrm{b}$ ).

Cette légalisation remettrait aussi en question les pratiques d'intervention actuelle qui ne sont pas volontaires, particulièrement celles effectuées sous le couvert de la justice. Si on ne cherche plus à définir les toxicomanies en rapport avec des normes juridiques où certains produits sont interdits, et que l'usage récréatif des drogues est reconnu, encore moins qu'auparavant pourra-t-on justifier le droit d'intervention sans le consentement de la personne? (CRD, 1985; Bertrand, 1981) Continuer d'obliger des personnes à se faire «traiter» sans leur consentement équivaut à croire que l'usage des produits est la cause des comportements jugés problématiques, et non l'effet de motivations plus profondes. De plus, criminaliser et «traiter» s'apparentent fortement lorsque le but majeur est la normalisation du sujet, mêrne si les moyens ne sont pas les mêmes (Vertongen, 1986; Nadeau, 1985; Ferracuti et Newman, 1972). Avec la légalisation des drogues, les modèles d'intervention devront être repensés en fonction de nouvelles définitions des problèmes, de leurs causes et du type d'aide qui correspond à l'approche des toxicomanies comme symptômes et non causes d'un déséquilibre (Beauchesne, 1987).

En résumé, nous sommes devant le tableau suivant: la légalisation des drogues, une meilleure éducation sur le sujet et des pratiques sociomédicales moins normatives pourraient contribuer à mieux prévenir les toxicomanies, à diminuer la répression et la stigmatisation et à faire en sorte que les appels à l'aide liés à la consommation de drogues soient mieux entendus et qu'on y réponde plus adéquatement. Mais un élément majeur manque à ce tableau: la nécessité de briser la logique économique actuelle dans la distribution des drogues.

\section{C) BRISER LA LOGIQUE DE RENTABILITÉ DANS LA DISTRIBUTION DES DROGUES}

Le système actuel de distribution de l'alcool n'est guère un modèle souhaitable pour la vente des drogues. La légalisation de ce produit 
sur le marché a permis, certes, d'assurer des garanties de qualité et une disponibilité adéquate pour ceux qui en font usage, mais sa commercialisation repose sur des stratégies de promotion qui en minimisent ou taisent les dangers pour en maximiser les ventes (Germain, 1984). Par exemple, on ne souligne pas les effets à court et à long terme de la consommation d'alcool avec les médications les plus courantes; pourtant, cette habitude de mélanger alcool et médicaments peut occasionner des dommages importants à la santé physique et mentale si on gère mal l'usage de ces produits.

La distribution actuelle des drogues prescrites - les médicaments -, est encore plus inadéquate comme modèle pour la vente des drogues dans une politique de santé. Théoriquement, la Loi sur les aliments et drogues sert à vérifier la qualité des produits et leur degré de nocivité afin que le public ait accès à de l'information adéquate et se procure des produits qui servent uniquement à sa santé. En pratique, toutefois, si on examine l'application de la Loi sur les aliments et drogues aux États-Unis - principale source des brevets pour les médicaments, et le rôle qu'y jouent les compagnies pharmaceutiques, on constate que le contrôle exercé sur les produits est quasi-nul car ces compagnies constituent de trop forts pouvoirs économiques (Sheskin, 1978; Témoignages et Dossiers, 1983; Brisson, 1986). En fait, l'application de cette loi, à l'heure actuelle, tend davantage à cautionner des pratiques dangereuses pour la santé publique mais bénéfiques aux profits des compagnies pharmaceutiques :

- des recherches expérimentales ne sont pas faites, sont faussées ou cachées;

- les médecins sont mal informés et même désinformés par les compagnies pharmaceutiques;

- les vendeurs de médicaments pour les hôpitaux, pharmacies ou autres travaillent généralement à commission et ont, ainsi, très peu d'intérêt à souligner les restrictions attachées à l'usage des médicaments;

- les étiquettes sur les médicaments ne précisent pas si un produit comporte des restrictions d'usage même si celles-ci sont connues;

- la publicité sur les médicaments n'est ni informative, ni éducative mais répète simplement le message qu'il faut fuir la douleur et être toujours serein grâce aux médicaments (Smart et Krakowski, 1973).

Et même lorsque des cas trop flagrants d'atteinte à la santé ont été révélés aux États-Unis, les membres du Bureau des aliments et drogues 
ont cherché davantage à les camoufler qu'à retirer les produits concernés du marché et à sanctionner les compagnies impliquées. La raison est toute simple: en plus des «cadeaux» faits au personnel chargé d'appliquer la Loi des aliments et drogues, celui-ci, le plus souvent, provient des compagnies pharmaceutiques et/ou vise à y retoumer (Sheskin, 1978; McNicoll, 1984; Témoignages et Dossiers, 1983).

Ainsi, à l'heure actuelle, le mode de distribution des médicaments répond à une logique de rentabilité qui engendre des milliers de morts, peu d'informations et d'éducation sur les produits, et des pratiques médicales «payées à l'acte» peu soucieuses de la santé publique. Cette politique de rentabilité étend ses tentacules sur le marché international; les pays du Tiers Monde, particulièrement, sont inondés de médicaments inadéquats et nocifs pour leur santé, eux qui sont accusés d'inonder les pays riches de stupéfiants. C'est un commerce bien artisanal comparativement à l'activité des compagnies pharmaceutiques (Témoignages et Dossiers, 1983; McNicoll, 1984).

C'est pourquoi il est nécessaire de briser la logique de rentabilité de la vente des drogues dans une optique de légalisation qui préserve un objectif de santé publique. Sinon, avec la légalisation, il est à parier que les compagnies pharmaceutiques commercialiseront toute une panoplie de pilules pour modifier les sens et aérer les esprits qui pourraient concurrencer de nouvelles drogues récréatives sur le marché; ces pilules, encore une fois, seront distribuées sans information, sans éducation adéquate du consommateur, et ne feront qu'accroître l'hécatombe liée aux mauvais usages de drogues.

\section{III. - L'ENJEU DE LA LÉGALISATION DES DROGUES}

La légalisation des drogues, à elle seule, résoudrait un certain nombre de problèmes secondaires liés à la criminalisation et au marché nơir, mais pourrait accroître les problèmes actuels liés à la commercialisation des drogues légales. Ce serait passer de Charybde en Scylla, du marché noir au marché institutionalisé, sans rien gagner dans la prévention des toxicomanies et en matière de santé.

Il est certain que la légalisation des drogues doit être envisagée pour abolir les répressions abusives qui camouflent les motifs liés à l'usage des drogues. Camoufler ces motifs permet trop souvent d'éviter les remises en question sur les piètres conditions de vie économiques, sociales, culturelles, environnementales, etc., dans lesquelles vivent bien des gens. 
Toutefois, il faut tenter d'imaginer la légalisation des drogues de manière à ce qu'elle ne serve pas à engraisser les logiques économiques qui sous-tendent actuellement nos politiques en matière de santé, politiques qui ont obtenu le cautionnement du savoir scientifique.

Dans cette perspective, la réflexion sur une politique future à l'égard des drogues ne fait que commencer, car elle draine bien des remises en question sur les normes qui régissent non seulement nos conditions de vie, mais nos vies elles-mêmes.

\section{BIBLIOGRAPHIE}

(1983), «La grande invasion: la pharmacie multinationale; la loi des plus forts», Témoignages et Dossiers, III(1), pp. 2-9.

BEAUCHESNE, Line (1988a), «La prévention de l'abus des drogues: question de contrôle social", in L'usage des drogues et la toxicomanie, Chicoutimi, Gaëtan Morin.

BEAUCHESNE, Line (1988b), «Les origines des lois canadiennes sur les drogues», in L'usage des drogues..., op.cit.

BEAUCHESNE, Line (1987), «La toxicomanie, une responsabilité de société», Psychotropes, IV(1), pp. 31-33.

BERTRAND, Marie-Andrée (1986a), «Permanence des effets pervers et résistance au changement des lois sur les drogues, Déviance et Société, X(3), pp. 177-191.

BERTRAND, Marie-Andrée (1986b), «Les intérêts professionnels : obstacles premiers aux changements des lois sur les drogues et à l'utilisation efficace des ressources pour toxicomanes», in Qualité de vie et drogues - Place aux jeunes, Chicoutimi, Gaëtan Morin, pp. 63-81.

BERTRAND, Marie-Andrée (1984), «Le contrôle de la drogue au Canada, dix ans après Le Dain», Le Devoir, mai.

BERTRAND, Marie-Andrée (1981), «Réflexions critiques sur le droit d'intervenir», Santé Mentale au Québec, VI(2), pp. 5-10.

BRISSON, Pierre et al. (1987), Le phénomène-drogue et les jeunes, Québec, ministère de l'Éducation.

BRISSON, Pierre (1986), «La toxicomanie aujourd'hui : aspects sociaux», in La toxicomanie, l'affaire de tout le monde, Québec, FOBAST, pp. 77-90.

COMMISSION LE DAIN (Commission d'enquête sur l'usage des drogues à des fins non-médicales) (1972), Rapport - Le cannabis, Ottawa.

COMMISSION LE DAIN (Commission d'enquête sur l'usage des drogues à des fins non-médicales) (1973), Rapport final, Ottawa.

CRD (Commission de réforme du droit) (1985), Protection de la vie - Les techniques de modification du comportement et le droit pénal, Document de travail 43, Ottawa, Information Canada. 
DAGOGNET, François (@1964, 1984), La raison et les remèdes, PUF.

ERICKSON, Patricia G. (1985), «Amérique du Nord. La réforme des lois sur le cannabis : un mouvement inachevé», Psychotropes, II(1), pp. 96-98.

ERICKSON, Patricia G. (1980), Cannabis Criminals, The Social Effects of Punishment on Drug Users, Toronto, ARF.

FERRACUTI, M.F. et G. NEWMAN (1972), «Perceptions clinique et psychologique de la déviance», in La perception de la déviance et de la criminalité, Vol. IX, Stratsbourg, Conseil de l'Europe.

GERMAIN, Georges-Hébert (1984), «Deux milliards de canettes à la recherche d'une nouvelle image», L'Actualité, février, pp. 53-56.

GLORIE, Jérôme (1984), «L'internationalisation des lois en matière de stupéfiants», Psychotropes, I(3), pp. 65-74.

GLORIE, Jérôme (1983), «Usage, commerce et contrôle de l'opium dans la Chine du 19ième siècle», Psychotropes, I(2), pp. 79-86.

HELMER, John (1975), Drugs and Minority Oppression, New York, The Seabury Press.

HERMANN, Kai et Horst RIECK (témoignages recueillis par) (@1978, 1981), Moi, Christiane F., 13 ans, droguée, prostituée..., Québec, Lacombe.

HULSMAN, L. et H. van RANSBEEK (1983), «Évaluation critique de la politique des drogues», Déviance et Société, III(3), pp. 271-280.

JONG (de), W. (1983), «Pour une véritable assistance aux consommateurs», Déviance et Société, III(3), pp. 281-286.

LAMARCHE, Pierre (1985), «Le trafic international des stupéfiants : variations sur le thème de l'échange inégal», Psychotropes, II(2), pp. 96-102.

LAPLANTE, Jacques (1974), «L'usager des drogues et les réglementations officielles», Toxicomanies, vol.7, pp. 177-195.

McNICOLL, André (1984), «Le commerce international des stupéfiants. Affaire de répression ou de développement", Psychotropes, I(3), pp. 75-80.

McNICOLL, André (1978), Procès de la décriminalisation du cannabis, Rapport spécial à l'intention des services de police, Ottawa. Polycopié.

NADEAU, Louise (1985), "Quand la communauté thérapeutique pour toxicomanes devient-elle maltraitante?», Santé Mentale au Québec, X(1), pp. 65-74.

RiCO, Jose M. (1986), «Les législations sur les drogues: origine et évolution", Psychotropes, III(1), pp. 69-83.

ROBINS, Lee N., John E. HELZER, Michi HESSELBROCK, Eric WISH (1980), "Vietnam veterans three years after Vietnam : how our study changed our view of heroin", in The Yearbook of Substance Use and Abuse, Vol.II, Leon Brill et Charles Winick (eds.), New York, Human Sciences Press, pp. 213-230.

SHESKIN, Arlene (1978), «Dangerous and unhealthy alliances: the pharmaceutical industry and the Food and Drug Administration», in The Evolution of Criminal Justice, Beverly Hills : Sage Publications, John P. Conrad (ed.), pp. 28-54.

SMART, R. G. et M. KRAKOWSKI (1973), «The Nature and frequency of drugs content in magazines and on television», Journal of Alcohol and Drug Education, 18, pp. 16-22. 
SMART, R.G. (1983), The Forbidden Highs, Toronto, ARF.

VALLEUR, Marc (1984), «Les conduites ordaliques chez les toxicomanes», Psychotropes, I(3), pp. 49-54.

VERTONGEN, Michel (1986), «Instances répressives et communautés thérapeutiques Faut-il accorder les violons?», Psychotropes, III(2), pp. 77-78.

ZAFIROPOULOS, Markos et Patrice PINELL (1982), «Drogues, déclassement et stratégies de disqualification», Actes de la recherche en sciences sociales, 42 (avril), pp. 61-75. 